Descargo de responsabilidad: las opiniones expresadas en los manuscritos son responsabilidad exclusiva de los autores. No necesariamente reflejan las opiniones de la editorial ni la de sus miembros.

\title{
Conducta desafiante preescolares de un centro educativo del departamento de Guatemala
}

\section{Oppositional defiant behavior Preschool of an Educational Center of the Department of Guatemala}

\author{
Alejandra Monzón Girón \\ alejandramonzon9003@postgradocunzac.edu.gt \\ https://orcid.org/0000-0002-8650-1038
}

Recibido: 19/11/2021

Publicado: 20/01/2022

\section{Filiación institucional de los autores}

Maestría en Neurociencia con énfasis en Neurocognición

Universidad de San Carlos de Guatemala
Ana Lucia Moscoso Figueroa

amoscoso@postgradocunzac.edu.gt https://orcid.org/0000-0003-3593-3874

Manuel Toledo Jacobo manueltoledo@postgradocunzac.edu.gt https://orcid.org/0000-0002-1271-6140

Nehemías Feliciano López Yes lopezyesnehemiasfeliciano@postgradocunzac.edu.gt https://orcid.org/0000-0002-3557-5133

\section{Referencia}

Monzón Girón , A., Moscoso Figueroa , A. L., Toledo Jacobo, M., \& López Yes, N. F. (2022). Conducta desafiante preescolares de un centro educativo del departamento de Guatemala. Revista Académica Sociedad Del Conocimiento Cunzac, 2(1), 49-55. DOI: https://doi.org/10.46780/sociedadcunzac.V2i1.12

\section{Resumen}

OBJETIVO: identificar factores que repercuten en la conducta desafiante de niños en la edad preescolar de un centro educativo del departamento de Guatemala. MÉTODO: se llevó a cabo un estudio descriptivo con 22 estudiantes en edad preescolar, entre ellos 12 de género femenino y 10 pertenecen al género masculino, permitió la recopilación de datos por medio de encuestas, observación y revisión de la literatura para fundamentar lo referente al trastorno negativista desafiante, esto con la finalidad de realizar la comprobación de los factores que afectan a los niños del preescolar. RESULTADOS: se evidencia, que el rango de género en la cual prevalece la conducta desafiante es el género masculino y las actitudes se manifiestan más en el contexto familiar de los niños. CONCLUSIÓN: se deduce que esta alteración de conducta se manifiesta en el ámbito familiar, con un desajuste emocional que da paso a conducta desafiante, por otro lado, se considera que los padres son permisivos, lo cual tienden a ser manipulados por los hijos ocasionando este déficit de emociones y de comportamiento.

\section{Palabras clave}

trastorno negativista desafiante, trastorno, conducta, preescolares 


\section{Abstract}

OBJECTIVE: to identify factors that affect the challenging behavior of children of preschool age of an educational center in the department of Guatemala. METHOD: a descriptive study was carried out with 22 preschool-age students, including 12 female and 10 males, it allowed the collection of data through surveys, observation and literature review to substantiate the reference to the Oppositional defiant disorder, this in order to verify the factors that affect preschool children. RESULTS: it is evident that the gender range in which the challenging behavior prevails is the male gender and the attitudes are manifested more in the family context of the children. CONCLUSION: it is deduced that this behavior alteration is manifested in the family environment, with an emotional imbalance that gives way to challenging behavior, on the other hand, it is considered that parents are permissive, which tend to be manipulated by the children causing this deficit of emotions and behavior.

\section{Keywords}

oppositional defiant behavior, disorder, conduct, preschool

\section{Introducción}

En este tiempo de confinamiento y de pandemia, muchos factores han desencadenado en el área emocional de los niños, llevándolos a conductas disruptivas. Un trastorno de conducta se define como una agrupación de síntomas que alteran la vida del niño en diferentes ámbitos como: social, familiar y escolar. Hoy en día con los avances de la psicología y de la neurociencia se logra comprender como la parte emocional afecta en el aprendizaje del niño, por lo que temas como trastorno negativista desafiante cobra bastante valor y no debe de dejarse pasar por alto.

No se sabe con exactitud qué es lo que hace que desencadene esta conducta, pero sí aseguran los expertos que el factor psicosocial tiene mucho que ver, como cuando hay una educación violenta, niños que se encuentran en un hogar temporal o crianza negligente, pueden estimular esta conducta. Es común notar conducta desafiante durante el desarrollo del niño en cualquier parámetro de edad, por lo que se debe de observar y verificar que no sea algo duradero, ya que entonces podría convertirse en un trastorno y afectar la vida del niño en sus diferentes ámbitos (Quicios, 2020).

El trastorno negativista desafiante, suele observarse en la etapa pre-escolar, llevando a que el niño tenga problemas notorios en su relación familiar y luego con sus compañeros, si esto no se trata a tiempo, se notará drásticamente un aumento en fracaso escolar, posterior a la delincuencia y problemas con la ley. De acuerdo a la revista Mayo Clínic que habla sobre el TND (2013), no sólo se trata de que el niño se porte mal, sino que manifiesta conductas a repetición, lo cual es confirmado por la Asociación Internacional de Psiquiatría, en su Manual de diagnóstico y estadístico del trastorno mental, quinta edición, hace mención que es un trastorno disruptivo, de control impulsivo y de conducta caracterizado por: enojo e irritabilidad, como un comportamiento desafiante argumentativo y espíritu de venganza (Quy, K. 2017).

El TND puede considerarse que tiene su origen desde la edad preescolar, cuando el niño o niña empieza a tener la capacidad de buscar cómo resolver sus instintos de curiosidad lo cual en ocasiones lo lleva a manifestar una conducta hostil, recurrente e inapropiado, principalmente hacia figuras de autoridad, afectando la relación hacia sus padres, entre sus hermanos o her- 
manas y otros familiares. Debido a que el TND presenta afecciones neurológicas, estudios han evidenciado que se debe a carencias de 5HT (serotonina) y norepinefrina (Armando, 2008). Estos estudios han demostrado que el TND tiene mayor incidencia en el género masculino, $3 \%$ en edades preescolares asociado a TDAH, datos que progresivamente aseguran que los síntomas se van afianzando hacia la edad escolar.

\section{Material y métodos}

Se llevó a cabo un estudio descriptivo con 22 estudiantes en edad preescolar, entre ellos 12 de género femenino y 10 pertenecen al género masculino, permitió la recopilación de datos por medio de encuestas, observación y revisión de la literatura para fundamentar lo referente al trastorno negativista desafiante, esto con la finalidad de realizar la comprobación de los factores que afectan a los niños de preescolar.

En la investigación se expresa los resultados adquiridos durante la entrevista a padres y docente del centro educativo, donde se considera los aspectos socioculturales como influyentes a desencadenar la tendencia de los preescolares a manifestar los trastornos conductuales que pueden ser característicos del trastorno negativista desafiante, por lo que se presenta la siguiente información.

Tabla 1. Conducta problemática de los preescolares con tendencia al TND.

\begin{tabular}{|c|c|c|c|c|}
\hline \multirow[t]{3}{*}{ Conducta DSM V } & \multicolumn{4}{|c|}{$\begin{array}{l}\text { Conducta hacia } \\
\text { los padres }\end{array}$} \\
\hline & \multicolumn{2}{|c|}{ SI } & \multicolumn{2}{|c|}{ No } \\
\hline & M & $\mathrm{F}$ & M & $\mathrm{F}$ \\
\hline A menudo pierde la calma. & 12 & 8 & 0 & 2 \\
\hline A menudo está susceptible o se molesta con facilidad. & 2 & 2 & 8 & 10 \\
\hline A menudo está enfadado y resentido. & 5 & 1 & 5 & 11 \\
\hline Discute a menudo con la autoridad o con los adultos. & 5 & 4 & 8 & 5 \\
\hline $\begin{array}{l}\text { A menudo desafía activamente o rechaza satisfacer la petición por parte } \\
\text { de figuras de autoridad o normas. }\end{array}$ & 5 & 3 & 5 & 9 \\
\hline A menudo molesta a los demás deliberadamente. & 1 & 0 & 9 & 12 \\
\hline A menudo culpa a los demás por sus errores o su mal comportamiento & 1 & 0 & 8 & 13 \\
\hline $\begin{array}{l}\text { Ha sido rencoroso o vengativo por lo menos dos veces en los últimos } \\
\text { seis meses. }\end{array}$ & 5 & 2 & 5 & 10 \\
\hline A menudo es rencoroso/a o vengativo/a & 1 & 0 & 9 & 12 \\
\hline
\end{tabular}

Fuente: elaboración propia. Con recolección de datos en encuesta. 
En la tabla 1 se hace manifiesta las diferentes conductas características del TND que comparado con estudios previos se afirma la literatura que expresa que es el género masculino quienes tienen mayor tendencia a manifestar estas conductas debido a ser por naturaleza más agresivos, fuertes e impulsivos, ya que son ellos quienes presentan tendencia a perder la calma y enfadarse rápidamente, desafiando a la figura de autoridad o a las normas establecidas en el salón de clase, comparado al género femenino quienes son más dóciles y sentimentales. Lo sobresaliente es que los preescolares en estudio manifiestan determinadas conductas hacia la figura de autoridad, en este caso expresada principalmente a los padres y docentes antes que expresarlos en otro tipo de ambiente social. La conducta que mayormente se observa es que los preescolares manifiestan rencor y venganza ante la negativa de sus solicitudes perdiendo con facilidad la calma y actuando de manera espontánea e impulsiva.

Tabla 2. Cambios conductuales en preescolares manifestadas en el salón según el entorno familiar.

\begin{tabular}{|c|c|c|c|c|c|c|c|c|c|c|c|}
\hline \multirow{3}{*}{$\begin{array}{l}\text { Cambios conductuales de preescolares en el } \\
\text { salón de clases }\end{array}$} & & & & & \multirow{3}{*}{$\begin{array}{c}\text { Frecuencia } \\
\text { semanal } \\
(1 \text { a 10) }\end{array}$} & \multirow{3}{*}{$\begin{array}{l}\text { Duración } \\
\text { aproximada } \\
\text { en minutos }\end{array}$} & \multirow{3}{*}{$\begin{array}{l}\text { Intensidad } \\
\quad(1 \text { a 10) }\end{array}$} & \multicolumn{4}{|c|}{ Padres } \\
\hline & \multicolumn{2}{|c|}{ SI } & \multicolumn{2}{|c|}{ No } & & & & $\mathbf{P}$ & A & C & $\mathrm{Se}$ \\
\hline & M & $\mathbf{F}$ & M & $\mathbf{F}$ & & & & & & & \\
\hline Desobedece reglas & 8 & 7 & 2 & 5 & 8 & 3 & 7 & $\mathrm{x}$ & & & $x$ \\
\hline Insiste pesadamente en una petición & 2 & 1 & 8 & 11 & 5 & 2 & 5 & $x$ & & $x$ & \\
\hline Daña o hiere a otros físicamente & 1 & 0 & 9 & 12 & 3 & 2 & 7 & $x$ & & & $x$ \\
\hline Destruye o rompe objetos a propósito & 1 & 1 & 9 & 11 & 1 & 1 & 3 & & $x$ & & $x$ \\
\hline Grita & 4 & 3 & 6 & 9 & 5 & 1 & 6 & $x$ & & $x$ & \\
\hline Llora con facilidad & 5 & 5 & 5 & 7 & 3 & 4 & 4 & $x$ & & $x$ & $x$ \\
\hline Se distrae fácilmente & 5 & 4 & 5 & 8 & 7 & 5 & 8 & $x$ & & $x$ & \\
\hline Demuestra entusiasmo y agrado de aprender & 10 & 12 & 0 & 0 & 9 & 7 & 8 & & $x$ & $x$ & \\
\hline No puede estar sentado tranquilo & 4 & 5 & 6 & 7 & 8 & 10 & 5 & $x$ & & $x$ & \\
\hline $\begin{array}{l}\text { Tiene dificultad para respetar la autoridad del } \\
\text { profesor }\end{array}$ & 3 & 2 & 7 & 10 & 5 & 10 & 2 & $x$ & & & $x$ \\
\hline Inicia peleas con sus compañeros & 1 & 1 & 9 & 11 & 2 & 0 & 2 & $x$ & & & $x$ \\
\hline Reacciona negativamente a la crítica y el fracaso & 4 & 5 & 6 & 7 & 3 & 2 & 6 & $x$ & $x$ & $x$ & \\
\hline No cumple reglas sin protestar & 6 & 8 & 4 & 4 & 4 & 3 & 4 & & $\mathrm{x}$ & $\mathrm{x}$ & \\
\hline
\end{tabular}

Fuente: elaboración propia. Con recolección de datos en encuesta.

En la tabla 2 se observan los resultados que describen las conductas más sobresalientes de los preescolares, asociados a las circunstancias producidas desde el hogar, en la sección de la derecha se expresa que los factores que predisponen a que los preescolares expresen conductas inadecuadas tienen su origen en el ambiente familiar, pues es allí donde el tipo de crianza generado por la manera en que el sistema de educación es implementado. Por ejemplo, los preescolares que desobedecen reglas tienen una estrecha relación con el estado emocional que los vincula a la situación sentimental entre los padres, generando el deseo de gritar, así 
como el hábito de tener dificultad en respetar las órdenes de los docentes, reaccionando negativamente a la crítica y a la sensación del fracaso, produciendo en los niños y las niñas el cumplimiento en última instancia de las órdenes con una desaprobación de insatisfacción

\section{Discusión}

Los niveles bajos de serotonina en el cerebro se asocian con niveles elevados de agresividad de tipo impulsiva. Personas con trastornos de comportamiento presentan déficits en el procesamiento de estímulos emocionales, sobre todo la angustia y el miedo. Por lo tanto, el lóbulo frontal es la última región del cerebro en madurar completamente por lo que las decisiones tomadas por los niños o adolescentes no habrá sido reflexionada. Asimismo, este trastorno es comorbido con otros trastornos, por lo que la comorbilidad más frecuente es el Trastorno por Déficit de Atención, lo cual es importante trabajar con un equipo multidisciplinario para evaluarlo.

Soutullo y Mardomingez (2010) argumentan que, para conseguir evitar los trastornos de conducta, primero se deben cambiar los distintos factores que están implícitos en el desarrollo de los mismos, como: factores biológicos, sociales, psicológicos, individuales y familiares. Por lo que tiene que ser una prioridad proteger a los necesitados y asegurar que los niños sean tratados con dignidad y que tengan oportunidades.

Los trastornos de conducta se han vuelto más comunes porque tienen varias fuentes de origen como familiar, la genética, el ambiente, la personalidad del niño, entre otros, también por la falta de importancia que las personas le aducen a los trastornos, en ocasiones se consideran berrinches, pero por el contrario, sin una correcta intervención se agravan conforme los niños y niñas crecen, se adhiere y puede convertirse en un trastorno de personalidad, y en el futuro en graves problemas.

Por lo que se considera que el factor determinante donde se da la aparición de estas conductas en la familia, se considera que en Guatemala se encuentra con mayor susceptibilidad en los niños estas conductas, puesto que en ocasiones el concepto familiar se ha devaluado debido a la violencia intrafamiliar, por situaciones laborales, la pobreza y sobre todo la pandemia que ha venido ocasionar un encierro de las familias lo cual permitió desatar emociones y actitudes violentas, negligentes en los niños y jóvenes, inclusive en padres de familia, por lo que es indispensable tratar a tiempo estas emociones para que no se conviertan en problemas graves en el futuro.

\section{Referencias}

Armando, S. H. (2008, 1 octubre). Oppositional Defiant Disorder. American Family Physician. Recuperado 22 de noviembre de 2021, de https://www.aafp.org/afp/2008/1001/p861.html

Quicios, B. (2020, 31 julio). Síndrome negativista desafiante de los niños, cuando los niños se muestran negativos, desobedientes y coléricos frente a sus padres. guiainfantil.com. Re- 
cuperado 8 de noviembre de 2021, de https://www.guiainfantil.com/articulos/educacion/ conducta/sindrome-negativista-desafiante-de-los-ninos/

Quy K, Stringaris A. (2017). Trastorno negativista desafiante. Irarrázaval M, Andres Martin (eds) Prieto-Tagle F, Gilibert Sánchez N (trad). En Rey JM (ed), Manual de Salud Mental Infantil y Adolescente de la IACAPAP. Ginebra: Asociación Internacional de Psiquiatría del Niño y el Adolescente y Profesiones Afines. https://iacapap.org/content/uploads/D.2-Oppositional-Defiant-Dis-Spanish-2017.pdf.

Trastorno negativista desafiante. (2013, 13 junio). Mayo Clinic. Recuperado 22 de noviembre de 2021, de http://ceril.net/index.php/articulos?id=140

Soutullo, C. y Mardominguez, J. (2010). Manual de psiquiatría del niño y adolescente. Buenos Aires, Argentina. Editorial médica panamericana. http://biblio3.url.edu.gt/Tesis/2015/05/42/ Lopez-Luisa.pdf

\section{Sobre los autores}

Grupo de investigadores de la Maestría en Neurociencias con énfasis en Neurocognición, Universidad de San Carlos de Guatemala.

Financiamiento de la investigación

Con recursos propios de los investigadores

\section{Declaración de intereses}

Declaran no tener ningún conflicto de intereses, que puedan haber influido en los resultados obtenidos o las interpretaciones propuestas.

\section{Declaración de consentimiento informado}

El estudio se realizó respetando el Código de ética y buenas prácticas editoriales de publicación. 


\section{Derechos de uso}

Copyright@ 2022 por Alejandra Monzón Girón, Ana Lucia Moscoso Figueroa, Manuel Toledo Jacobo y Nehemías Feliciano López Yes.

Este texto está protegido por una licencia Creative Commons 4.0

Este texto está protegido por la Licencia Creative Commons Atribución 4.0 Internacional.

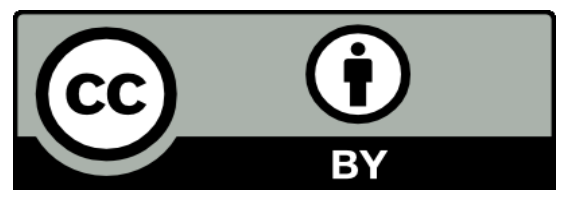

Este texto está protegido por una licencia

Creative Commons 4.0.

Es libre para compartir, copiar y redistribuir el material en cualquier medio o formato y adaptar el documento, remezclar, transformar y crear a partir del material para cualquier propósito, incluso comercialmente, siempre que cumpla la condición de atribución: debe reconocer el crédito de una obra de manera adecuada, proporcionar un enlace a la licencia, e indicar si se han realizado cambios. Puede hacerlo en cualquier forma razonable, pero no de forma tal que sugiera que tiene el apoyo del licenciante o lo recibe por el uso que hace. 